\title{
Criminologie
}

\section{Les signaux précoces de l'agir délinquant fréquent}

\section{David P. Farrington}

Volume 19, numéro 2, 1986

La prédiction de la carrière criminelle

URI : https://id.erudit.org/iderudit/017238ar

DOI : https://doi.org/10.7202/017238ar

Aller au sommaire du numéro

Éditeur(s)

Les Presses de l'Université de Montréal

ISSN

0316-0041 (imprimé)

1492-1367 (numérique)

Découvrir la revue

Citer cet article

Farrington, D. P. (1986). Les signaux précoces de l'agir délinquant fréquent. Criminologie, 19(2), 9-31. https://doi.org/10.7202/017238ar

\section{Résumé de l'article}

A great deal is known about the early precursors of offending in general, but much less is known about early factors which discriminate between high rate and low rate offenders. Childhood antisocial behaviour, criminal parents and siblings, indicators of social deprivation such as low income, and low intelligence and attainment predict both offending and high rate offending. Many of the future chronic offenders could have been predicted by these factors at the time of their first conviction. However, child rearing factors such as poor parental supervision, harsh or erratic parental discipline, and parental conflict are excellent predictors of offending in general but not of high rate as opposed to low rate offending. Little is known about protective factors which might prevent high risk children from becoming high rate offenders.
Ce document est protégé par la loi sur le droit d'auteur. L'utilisation des services d'Érudit (y compris la reproduction) est assujettie à sa politique d'utilisation que vous pouvez consulter en ligne.

https://apropos.erudit.org/fr/usagers/politique-dutilisation/ 
A great deal is known about the early precursors of offending in general, but much less is known about early factors which discriminate between high rate and low rate offenders. Childhood antisocial behaviour, criminal parents and siblings, indicators of social deprivation such as low income, and low intelligence and attainment predict both offending and high rate offending. Many of the future chronic offenders could have been predicted by these factors at the time of their first conviction. However, child rearing factors such as poor parental supervision, harsh or erratic parental discipline, and parental conflict are excellent predictors of offending in general but not of high rate as opposed to low rate offending. Little is known about protective factors which might prevent high risk children from becoming high rate offenders.

\section{L'AGIR DÉLINQUANT}

\subsection{DÉFINITION ET MESURE}

Un des résultats, majeur, de l'étude de Wolfgang $e t$ al. (1972), à Philadelphie, d'une cohorte de 10000 garçons nés en 1945 , était que $6 \%$ de l'échantillon était responsable de $52 \%$ de la délinquance juvénile consignée. Ces $6 \%$, dits délinquants chroniques, avaient subi au moins cinq arrestations chacun. Ils représentaient $18 \%$ des auteurs de délits mais avaient à leur crédit un pourcentage substantiel des délits graves : $63 \%$ de l'ensemble des délits de l'index du Uniform Crime Report, $71 \%$ des homicides, $73 \%$ des viols, $82 \%$ des vols qualifiés et $69 \%$ des assauts graves. Des résultats semblables furent obtenus auprès d'une seconde cohorte masculine, née en 1958. Les délinquants chroniques, $7,5 \%$ de la cohorte et $23 \%$ des auteurs de délits, avaient à leur actif $61 \%$ de tous les délits, $69 \%$ de ceux de l'index, $61 \%$ des homicides, $76 \%$ des viols, $73 \%$ des vols qualifiés et $65 \%$ des assauts graves (Wolfgang et Tracy,

* Traduit de l'anglais par Suzanne Laflamme-Cusson.

** L'auteur est professeur à l'Institut de criminologie de l'Université de Cambridge, Angleterre.

Criminologie, 19,2, 1986 
1982). Il ressort clairement de ces études, comme d'autres études aussi, que la plupart des délinquants persistants ont aussi tendance à s'adonner aux délits les plus graves.

D'autres chercheurs, en divers lieux, ont aussi constaté qu'une petite fraction de personnes commettent une large part de tous les délits. À Racine, Wisconsin, Shannon (1978) a trouvé que $7 \%$ d'une cohorte de 1300 enfants nés en 1942 avaient fait l'objet de $51 \%$ de tous les rapports policiers relatifs à la cohorte en 32 ans et que $7 \%$ d'une cohorte de 2100 enfants nés en 1949 étaient responsables de $52 \%$ de tous les contacts policiers survenus au cours de leurs vingt-cinq premières années.

À Londres, Farrington (1983) rapporte que $6 \%$ d'un échantillon de 400 garçons ( $17 \%$ de tous les auteurs de délits) avaient à leur actif $49 \%$ de toutes les condamnations criminelles prononcées avant 25 ans. Ils avaient tous été reconnus coupables de délits au moins six fois. Dans un échantillon national de garçons anglais et gallois nés en 1953, les 5,5\% qui avaient au moins six condamnations chacun (18\% des auteurs de délits) s'étaient avérés coupables de $70 \%$ de toutes les affaires élucidées jusqu'à leur $28^{\mathrm{e}}$ année (Home Office, 1985).

Tous ces résultats ont été obtenus à partir des registres officiels de délits. Néanmoins, les délinquants chroniques officiels dans l'étude londonienne avaient aussi à leur actif des proportions substantielles de tous les délits révélés (lors de sondages) par l'échantillon : entre 15 et 18 ans, ces $6 \%$ avaient commis $30 \%$ de tous les cambriolages, $32 \%$ des vols de véhicules, $24 \%$ des vols de magasins et $21 \%$ des vols dans les voitures (Blumstein et al., 1985). Cette étude révélait aussi une remarquable concentration de la délinquance dans un petit nombre de familles. West et Farrington (1977) ont trouvé que seulement $4,5 \%$ de toutes les familles avaient fait l'objet de près de la moitié ( $48 \%$ ) des condamnations criminelles de tous leurs membres réunis (pères, mères, fils, filles).

Les études de délinquance révélée nous apprennent aussi l'existence de grandes variations dans la fréquence de l'agir délinquant. Dans une recherche de la Rand Corporation sur des détenus, Greenwood et Abrahamse (1982) ont constaté que si leurs sujets avaient commis des cambriolages à une fréquence médiane de 5,5 par année, les $10 \%$ plus actifs des cambrioleurs s'y étaient adonnés à une fréquence supérieure à 230 par an. 
Dans une autre étude de la Rand Corporation, où des détenus californiens remplissaient des questionnaires de délinquance révélée, Peterson et al. (1981) ont trouvé que le sujet moyen avait commis 14 crimes graves (homicide, viol, vol qualifié, cambriolage, assaut, vol d'auto et usage de faux) par année de liberté mais que les $8 \%$ plus actifs en avaient commis au-delà de 60 . Ces $8 \%$ étaient à eux seuls responsables de trois fois plus de crimes graves que la somme des mêmes crimes commis par la moitié la moins active des prisonniers. Il est clair que, tandis que la majorité des auteurs de délits en commettent relativement peu fréquemment, un petit nombre de délinquants persistants est très prolifique.

Si seulement on était capable d'identifier ces délinquants très productifs au début de leur carrière et si on pouvait réduire leur rythme (à zéro de préférence) par des méthodes préventives, neutralisantes, dissuasives ou réhabilitantes, cela nous mènerait vraisemblablement à une diminution significative du total des crimes commis. Comme premier pas vers une telle identification précoce, cet article relate ce qu'on connaît des signaux de la délinquance à haute fréquence.

\subsection{PEUT-ON PRÉDIRE L'AGIR DÉLINQUANT FRÉQUENT?}

Blumstein et Moitra (1980) ont émis de sérieuses réserves quant au concept de délinquant chronique identifiable. Étant donné, disent-ils, que toutes les distributions statistiques de fréquence de l'agir délinquant individuel permettent de constater l'existence de délinquants relativement prolifiques, on doit seulement s'attendre à ce que ces délinquants soient responsables d'une portion relativement élevée de l'ensemble des délits. Même si tous les jeunes ayant fait l'objet d'arrestation(s), dans l'étude de cohorte de Philadelphie, avaient eu un penchant criminel identique, le hasard à lui seul aurait fait que certains auraient été arrêtés plus souvent que d'autres. Blumstein et Moitra ont montré qu'en postulant des probabilités de récidive identiques chez tous les sujets arrêtés au moins trois fois, on était assez proche des distributions de fréquence d'arrestations observêes. Dès lors on aurait pu au départ être dans l'impossibilité de distinguer entre ceux qui se sont avérés délinquants chroniques (cinq arrestations ou plus) et ceux qui ont changé de cap après trois ou quatre arrestations. La question cruciale, 
pour la théorie comme pour la politique criminelle, n'est pas de savoir si une petite fraction des délinquants est responsable d'une grande portion des délits (c'est dans une large mesure inéluctable) mais de savoir si les délinquants chroniques, de quelle que manière qu'on les définisse, peuvent être identifiés avant qu'ils le deviennent.

La première occasion d'intervenir dans le système de justice criminelle est soit la première arrestation, soit la première déclaration de culpabilité. C'est un moment critique pour identifier les délinquants virtuellement chroniques. C'est pourquoi un des objectifs de cet article est de passer en revue ce qu'on connaît des signaux de la délinquance fréquente, au moment de ce premier contact officiel consigné.

Malheureusement, Dunford et Elliott (1984), dans leur étude suivie d'un échantillon représentatif national de $1700 \mathrm{mi}-$ neurs, ont affirmé que les plus engagés dans la carrière délinquante ne sont jamais arrêtés. Si c'était vrai, on ne pourrait trop compter sur des interventions judiciaires pour barrer la route aux auteurs de délits qui s'annoncent prolifiques. Dunford et Elliott ont appelé délinquants graves persistants (serious career offenders) les auteurs d'au moins trois délits de l'index (révélés par questionnaire), et ce au moins deux ans de suite, sur les cinq de leur étude. Seul environ le quart de ces délinquants avaient été appréhendés et un peu moins du quart, dans le cas des auteurs de dix délits et plus de l'index. Chez les plus prolifiques de tous (qui avouaient au moins vingt délits de l'index), seulement un délit sur quarante avait mené à une arrestation.

On peut s'objecter aux arguments de Dunford et Elliott. Premièrement, de soi-disant délits de l'index n'auraient peutêtre pas été vus du même œil par la police. Il était souvent question de violence envers les élèves, professeurs ou autres, de batailles de gang et d'assauts. Deuxièmement, vu que $27 \%$ de l'échantillon n'a pas été interviewé, les auteurs des délits les plus graves et les délinquants les plus engagés peuvent avoir été sous-représentés dans leur échantillon. Il ressort donc que leurs déiinquants graves persistants de carrière supportent mal la comparaison avec les chroniques de Philadelphie. Troisièmement, les données policières sur les arrestations peuvent être incomplètes. On a recherché que les dossiers des jeunes qui y 
avaient consenti $(88 \%)$ et seulement dans un rayon de dix milles de la résidence des jeunes. Et puis on ne retenait que les arrestations colligées depuis trois ans plutôt que de couvrir toute l'histoire juvénile.

À l'opposé, l'étude longitudinale de West et Farrington (1977), à Londres, portait sur un échantillon des jeunes d'une seule ville dont $6 \%$ avaient été reconnus coupables d'au moins six crimes avant l'âge de 25 ans. Parmi eux, $95 \%$ furent interviewés à 18 ans (une plus grande fraction l'avait été aux étapes antérieures) et les analyses de fréquence de leurs délits portaient sur des infractions criminelles à la gravité bien établie comme les cambriolages. West et Farrington ont trouvé que la majorité de ceux qui avaient admis s'être adonné au cambriolage, sur une période de trois ans, en avaient aussi été accusés au cours de cette période. Seulement $13 \%$ des cambriolages avaient abouti à des condamnations mais la plupart des cambrioleurs avaient été arrêtés un jour ou l'autre. À peu près la moitié des auteurs d'un premier cambriolage avaient été arrêtés, comparativement à $58 \%$ de ceux qui en avaient commis entre deux et cinq et à $77 \%$ pour six et plus. Le fait que les augmentations de pourcentage ne soient pas plus spectaculaires suggère l'idée que les délinquants les plus prolifiques s'y prennent de manière moins risquée que les occasionnels. Farrington (1983) a conclu que les données de délinquance officielle et révélée s'accordent assez bien pour identifier les cambrioleurs, même si les deux méthodes s'écartent grandement dans leurs estimations du nombre des cambriolages commis.

Considérant un plus large éventail de délits, West et Farrington ont aussi constaté une grande concordance entre délinquance révélée et officielle. Des 80 garçons (sur les 409 interviewés) qui avaient avoué le plus d'actes délinquants, à quatorze et seize ans combinés, 22 ont été reconnus coupables à plusieurs reprises, 19 l'ont été une fois, 13 avaient un dossier policier pour autre chose (délit mineur ou avertissement de la police) et seulement 26 n'avaient aucun dossier. La majorité des récidivistes (22 sur 37) appartenaient au groupe des délinquants prolifiques au test de délinquance révélée. Par contre, des 93 garçons ayant avoué seulement un petit nombre d'actes, dont aucun délit grave, pas un seul n'était officiellement récidiviste, seulement cinq avaient une condamnation comme mineurs, deux avaient un autre dossier à la police et 86 n'avaient rien de consigné. Il 
ressort de ces résultats que les délinquants qui s'avouent très actifs dans les questionnaires recouvrent grandement ceux qui le sont d'après les dossiers officiels.

Le but de cet exposé est de résumer les connaissances acquises sur les caractéristiques, au moment du premier contact policier ou judiciaire, ou antérieurement à lui, propres à ceux qui commettront des délits à une fréquence élevée en tant que mineurs ou jeunes adultes.

\subsection{MÉTHODES DE RECHERCHE}

Il est clair qu'on a besoin d'études longitudinales «prospectives» pour déterminer jusqu'à quel point il est possible de prédire la délinquance fréquente (pour une revue de ces études, voir Farrington, 1979b). Même lorsqu'on a fait des études longitudinales, elles n'ont habituellement pas porté sur les signes avant-coureurs de la délinquance fréquente ni sur l'identification, parmi tous les auteurs de délits, de ceux qui commettraient les plus graves et avec le plus de persistance. Plusieurs études de délinquance officielle nous ont renseigné sur des différences précoces entre délinquants et non-délinquants, contrairement à la plupart des études de délinquance révélée. D'où on connaît davantage les signaux précoces de la délinquance par rapport à des mesures d'arrestation ou de condamnation que par rapport à des mesures de délinquance révélée. L'excellente recension des écrits scientifiques de Loeber et Dishion (1983) montre que les meilleurs éléments prédicteurs de l'apparition de la délinquance (s'il y aura ou non commission de délit) sont, dans l'ordre, la discipline familiale, les troubles de comportement enfantins tels le vol, le mensonge et l'école buissonière; la criminalité ou la conduite antisociale des autres membres de la famille, une piètre performance scolaire, le fait de vivre séparé de ses parents et le statut socio-économique. Nous discuterons plus loin de l'importance de tous ces facteurs par rapport à la délinquance fréquente.

L'information la plus pertinente pour mon propos provient des études longitudinales comparant non-délinquants, délinquants non chroniques et délinquants chroniques. Cette classification permet d'étudier les signes précurseurs de la délinquance (versus non délinquance) et ceux de la délinquance chronique (versus non chronique). Malheureusement, il existe peu d'études de ce genre. Wolfgang et al. (1972) ont comparé ces trois 
groupes dans la toute première étude de cohorte, qu'ils ont réalisée à Philadelphie, mais leurs conclusions étaient limitées à cause de la pénurie d'informations sur leur échantillon dans les registres.

Blumstein et al. (1985) ont aussi comparé ces trois groupes en utilisant un éventail beaucoup plus large de variables dans leur étude longitudinale à Londres et purent ainsi scruter en détail les signaux précoces de la délinquance chronique. D'autres comparaisons pertinentes ont été rapportées par West et Farrington (1977), notamment entre les jeunes délinquants qui devinrent des délinquants adultes et ceux qui ne le devinrent pas et entre les récidivistes officiels de dix-neuf ans qui ont été condamnés ultérieurement et ceux qui ne l'ont pas été.

\section{LES SIGNAUX PRÉCOCES}

\subsection{LE COMPORTEMENT ANTISOCIAL}

Comme Loeber et Dishion l'ont conclu de leur examen des recherches réalisées, les comportements insupportables, malhonnêtes, agressifs et antisociaux de l'enfance ont toujours été considérés comme d'importants préludes de la délinquance ultérieure. Par exemple, Emsminger et al. (1983) ont montré que, chez les enfants d'un ghetto noir urbain de Chicago, les scores d'agressivité attribués par les instituteurs à leurs élèves de première année (six ans) étaient en relation significative avec les scores de délinquance révélée des mêmes jeunes à quinze ans. De même, concernant une zone à forte délinquance de la ville de New York, Craig et Glick (1968) ont dit que les problèmes de comportement en première année laissent prévoir une délinquance grave et persistante ultérieure.

A Londres, Farrington (1978) a découvert que des scores d'agressivité attribués par les instituteurs à leurs élèves de huit ans permettaient de prédire non seulement l'agir violent révélé à dix-huit ans, mais aussi les condamnations pour des crimes de violence. Dans une étude longitudinale sur les enfants anglais nés en 1946, Mulligan et al. (1963) ont montré que les garçons jugés coupables avaient été décrits par leurs professeurs comme agressifs, querelleurs, désobéissants, rebelles à la critique, menteurs, tricheurs et manquant l'école sans raison. De la même manière, dans une étude longitudinale en Suède, Magnusson 
et al. (1975) ont rapporté que les estimés de l'agressivité faits par les instituteurs permettaient de prédire avec assez d'à-propos les futurs démêlés avec la justice des mineurs.

La démonstration la plus troublante de la continuité dans l'agir antisocial entre l'enfance et l'âge adulte nous est fournie par Robins $(1979,1983)$, qui a défendu l'idée que les écarts de conduite dans l'enfance et la personnalité antisociale adulte sont liés dans leur développement, que ces syndrômes adhèrent en quelque sorte l'un à l'autre. Dans son étude de relance de trente ans, sur 500 enfants menés à une clinique d'aide à l'enfance à Saint-Louis (Robins, 1966), elle a trouvé que ces enfants volaient, faisaient l'école buissonnière, fuyaient la maison, étaient agressifs, énurétiques, indisciplinés à l'école, menteurs pathologiques et le reste. Parvenus à l'âge adulte, ils avaient tendance à être arrêtés, internés en psychiatrie, alcooliques, dévoyés sexuellement, divorcés, errants, mauvais payeurs, piètres travailleurs et ainsi de suite. En général, les adultes chez qui on avait diagnostiqué une personnalité antisociale avaient presque tous été des enfants antisociaux, même si plusieurs. enfants antisociaux n'étaient pas demeurés tels à l'âge adulte.

Robins et Ratcliff (1980) ont exploré les relations entre neuf types de comportements déviants de l'enfance et cinq patterns d'arrestation adulte. Ils ont trouvé que la probabilité d'être arrêté pour un adulte augmentait avec le nombre de types de comportements problèmes présentés dans l'enfance. Ils ont poussé plus loin pour voir si des types spécifiques d'actes de l'enfance permettaient de prédire des patterns spécifiques d'arrestations comme adulte, en contrôlant le nombre des uns et des autres, mais ils ne trouvèrent de relation significative que pour la consommation de drogue. Robins en a logiquement déduit que, puisque les relations les plus fortes dans sa recherche sont de l'ordre de la quantité plutôt que de la qualité (le niveau global de comportement antisocial adulte), il s'agit d'un seul et unique syndrôme constitué d'une grande variété d'actes antisociaux, qui apparaissent dans l'enfance et se poursuivent à l'âge adulte.

Dans l'étude longitudinale de Londres, Farrington (1979a) a montré que la tendance à être insupportable, la malhonnêteté et la témérité entre huit et dix ans, évaluées par les instituteurs, les pairs et les parents annoncent réellement la délinquance officielle aussi bien que révélée, juvénile autant qu'adulte. 
Farrington (1985b) a scruté les préludes à la délinquance officielle et révélée aux différents âges. Le meilleur élément prédicteur de délinquance, à tout âge, était la mesure de la délinquance à l'âge immédiatement précédent, ce qui montre la continuité dans le temps de la criminalité. Le meilleur prédicteur de la délinquance de vingt-et-un à vingt-cinq ans était celle de dix-sept à vingt ans et ainsi de suite pour quatorze à seize ans et dix à treize ans. La délinquance de ce dernier intervalle d'âges avait comme meilleur prédicteur les problèmes de comportement de huit à dix ans. Il a semblé y avoir une très réelle continuité entre les problèmes de comportement de l'enfance et la conduite criminelle adulte.

Blumstein et al. (1985) ne nous disent pas seulement à quel point divers facteurs prédisent la délinquance (versus la nondélinquance) mais aussi combien ils permettent d'identifier les délinquants chroniques parmi l'ensemble des auteurs de délits. Les problèmes de comportement étaient un prédicteur appréciable quant à ces deux dimensions et les relations observées étaient indépendantes des autres éléments de prédiction. Dès lors, on peut conclure que le comportement antisocial avant dix ans permet de prédire non seulement la délinquance ultérieure en général mais aussi la délinquance à haute fréquence.

\subsection{LE MILIEU FAMILIAL}

Comme nous l'avons mentionné, Loeber et Dishion (1983) ont conclu de leur recension des écrits scientifiques que la discipline familiale, la délinquance des parents et de la fratrie, la séparation d'avec les parents et le statut socio-économique de la famille permettaient de prédire la délinquance. Voyons maintenant de plus près chacun de ces signaux précoces.

McCord (1979) a réalisé une analyse fort intéressante des signes avant-coureurs précoces de la délinquance dans son étude follow-up de trente ans sur 250 garçons traités (par counseling) en vertu du programme de Cambridge-Somerville. Elle a trouvé qu'une surveillance défaillante des parents était le meilleur prédicteur des crimes ultérieurs aussi bien contre la personne que contre les biens. L'agressivité parentale (incluant une discipline cassante) et le conflit entre les parents étaient des signes annonciateurs de crimes contre la personne mais non de crimes contre les biens et inversement pour l'attitude mater- 
nelle (passive ou de rejet). Robins (1979), dans ses longues études follow-up a aussi trouvé qu'une surveillance et une discipline médiocres étaient également des signaux précoces logiques d'un comportement criminel ultérieur.

West et Farrington (1973) ont obtenu des résultats semblables à Londres. Une discipline trop rude ou erratique; la cruauté, la passivité ou la négligence dans les attitudes parentales; une piètre surveillance, ou un conflit entre les parents : tous ces éléments, mesurés à huit ans, étaient prédicteurs de délinquance officielle ultérieure. Qui plus est, de mauvaises méthodes d'éducation des parents (discipline, attitudes et conflit combinés) et une piètre surveillance annonçaient la délinquance autant révélée qu'officielle (Farrington, 1979a). La dureté dans la discipline et les attitudes, mesurée auprès des enfants de huit ans, laissait prévoir l'émergence de la violence (versus les délits non violents) (Farrington, 1978). Mais de piètres méthodes éducatives étaient reliées à la délinquance précoce seulement (Farrington, 1985b); elles n'apparaissaient pas chez ceux qui ne furent jugés coupables qu'une fois adultes (West et Farrington, 1977). Dès lors, il n'est pas étonnant que la discipline et la surveillance exercées au cours du jeune âge ne permettent pas de prédire de manière significative le caractère chronique ou temporaire de la délinquance (Blumstein et al., 1985).

Dans son étude longitudinale, Robins (1979) a décelé une tendance, chez les enfants de parents antisociaux ou alcooliques, à devenir criminels. Par exemple, dans son étude follow-up de 200 hommes noirs de Saint-Louis, elle a montré que les parents (ses sujets) qui avaient subi une arrestation avaient souvent des enfants qui en subissaient aussi et que les dossiers juvéniles des uns et des autres se ressemblaient en termes de fréquence et de types de délits (Robins et al., 1975). McCord (1977) dans l'étude de Cambridge-Somerville, a aussi rapporté que les enfants (ses sujets) jugés coupables étaient souvent issus de pères qui l'avaient été aussi. Son étude ne montre pas clairement s'il y a semblable relation entre les motifs respectifs de condamnation. McCord a trouvé que $29 \%$ des pères coupables de violence avaient des enfants également coupables de violence, contre seulement $12 \%$ des autres pères. Mais cela peut ne refléter que la tendance générale pères coupables - fils coupables plutôt qu'une tendance spécifique pères violents - fils violents. Craig et Glick (1968) à New York ont aussi montré que les garçons 
ayant des parents, frères ou sœurs criminels avaient une plus grande propension à présenter une délinquance lourde et fréquente.

Dans l'étude longitudinale de Londres, West et Farrington (1973) ont trouvé que le fait pour un garçon, le jour de ses dix ans, d'avoir une mère, un père et un frère reconnus coupables prédisait de manière significative sa propre culpabilité ultérieure. De plus, le fait d'avoir des parents jugés coupables et quelqu'un de la fratrie délinquant était relié à la délinquance tant révélée qu'officielle (Farrington, 1979a). Contrairement à la plupart des autres prédicteurs, la criminalité des parents prédisait moins l'engagement précoce dans la délinquance qu'une délinquance plus tardive (Farrington, 1985b). De plus, ce facteur signalait lesquels parmi les jeunes délinquants deviendraient des criminels adultes et quels récidivistes de dix-neuf ans continueraient encore dans la voie de la délinquance (West et Farrington, 1977). Tandis que la criminalité des parents était le meilleur prédicteur indépendant de la présence de l'agir délinquant, la condamnation d'un membre de la fratrie s'avérait le meilleur prédicteur indépendant de sa chronicité (Blumstein et al., 1985). Les facteurs «parent reconnu coupable» et «membre de la fratrie reconnu coupable» ayant tendance à être reliés, lorsque l'un des deux ressortait comme prédicteur indépendant, c'était, bien entendu, à l'exclusion de l'autre.

McCord (1982) a réalisé une étude qui retient l'attention sur la relation entre le bris du foyer (par la perte du père naturel) et la délinquance grave ultérieure. Elle a décelé une haute fréquence de délinquance chez les garçons élevés, dans un foyer brisé, par une mère peu affectueuse ou, dans un foyer intact, par des parents en conflit. Les combinaisons foyer brisé + mère affectueuse et foyer intact + absence de conflit étaient associées à une délinquance peu fréquente. Ces résultats nous portent à croire que ce n'est pas tant la rupture du foyer qui est criminogène que le conflit des parents qui y a mené. Des conclusions semblables ressortent de l'étude longitudinale nationale anglaise (Wadsworth, 1979). Les garçons issus de foyers brisés par le divorce ou la séparation des parents ont une plus grande probabilité de délinquance que ceux dont le foyer a été brisé par un décès. Il n'est pas sans intérêt d'apprendre que le remariage était aussi relié à un risque accru de délinquance, ce qui nous 
autorise à croire que les familles monoparentales pourraient être moins criminogènes que celles où deux parents sont en conflit.

Power et al. (1974) ont cherché jusqu'à quel point l'appartenance à un foyer brisé permettait de prédire la récidive chez des garçons londoniens âgés de onze à quatorze ans lors de leur première comparution en cour. Comme dans les autres recherches, il s'est avéré que les garçons issus de foyers brisés par un décès n'avaient pas plus tendance à récidiver que les garçons issus de familles intactes sans stress. Toutefois, les garçons issus de familles brisées par suite du désaccord des parents avaient un haut taux de récidive, tout comme les garçons issus de foyers intacts aux prises avec un stress grave et persistant.

West et Farrington (1975) ont aussi trouvé que les garçons orphelins n'avaient pas particulièrement tendance à être trouvés coupables de délits, contrairement à ceux qui appartenaient à des foyers brisés par la séparation ou la désertion de leurs parents. Aussi, ils en sont venus à la conclusion que le fait d'avoir un foyer brisé en bas âge n'était pas spécialement criminogène. Qu'elle s'avère permanente ou non, pourvu qu'elle dure plus d'un mois, la séparation est un élément prédicteur de délinquance officielle, sauf lorsqu'elle est attribuable au décès ou à l'hospitalisation. En outre, une telle séparation est en relation avec la délinquance révélée autant qu'officielle (Farrington, 1979a). Néanmoins, cette variable ne permet pas de prédire entre les formes chroniques et non chroniques de la délinquance (Blumstein et al., 1985).

La dernière caractéristique familiale que nous aborderons ici est la carence au plan social dont les indices retenus sont la pauvreté, la dépendance de l'aide sociale et l'emploi peu valorisé des parents. Comme Thornberry et Farnworth (1982) l'ont souligné, l'abondante littérature sur les rapports entre la classe sociale et le crime est pleine d'inconsistances. Quoi qu'il en soit, la plupart des études sur la question étant essentiellement de nature corrélative, elles ne cherchent pas si la classe sociale est un élément prédicteur de la délinquance. De plus, la plupart des recherches existantes mesurent la classe sociale par le biais du lieu de résidence plutôt que par des caractéristiques familiales (ce qui nous intéresse ici). En aucun cas, le statut socioéconomique basé sur le prestige occupationnel ne peut être un bon indicateur de carence sociale. 
Les études longitudinales tendent à montrer, avec plus de consistance que toutes les autres approches, que les enfants issus de parents de classe inférieure et particulièrement de parents qui souffrent de privations sociales, ont plus tendance à devenir délinquants en grandissant. Par exemple, Robins (1979) nous rapporte, à partir de deux relances, que les enfants de familles pauvres, vivant dans les bas quartiers et dont les parents ou gardiens ont un bas statut occupationnel, deviennent plus souvent délinquants. McCord (1979) pour sa part n'a pas constaté qu'un emploi peu valorisé était un élément précurseur de délinquance officielle dans son échantillon. Toutefois, dans l'étude longitudinale nationale anglaise, Douglas et al. (1966) ont montré que la fréquence de l'agir délinquant variait selon le statut occupationnel des parents, passant de $3 \%$ aux plus hauts échelons sociaux à $19 \%$ aux plus bas. La recherche de Van Dusen et al. (1983) auprès des enfants adoptés au Danemark est intéressante en ce qu'elle montre que le prestige occupationnel des parents biologiques comme adoptifs avait une valeur prédictive de la délinquance déclarée ultérieure des enfants.

Dans leur recherche longitudinale à Londres, West et Farrington (1973) ont trouvé qu'un bas revenu familial, un logement inadéquat et l'inconstance professionnelle du père aidaient à prédire la délinquance officielle, mais qu'il n'en était rien quant au prestique occupationnel du pourvoyeur de la famille. Toutefois, tous ces facteurs permettaient de prédire la délinquance juvénile révélée par questionnaire aussi bien que la délinquance officielle adulte (Farrington, 1979a). De plus, un bas revenu familial était le facteur le plus discriminant entre délinquants chroniques et non chroniques (Blumstein et al., 1985).

En conclusion de cette partie, une piètre surveillance des parents, une discipline cassante et inconstante et un foyer brisé par suite d'un conflit entre les parents sont tous des signaux importants de la délinquance précoce. S'ils permettent probablement de discriminer entre les délinquants prolifiques et le reste d'une population, ils ne semblent pas distinguer ceux qui auront un haut niveau de délinquance de l'ensemble des auteurs de délits. Par contre, la criminalité des autres membres de la famille et des indices de carence au plan social permettent de prévoir non seulement quels éléments d'une population normale commettront des délits mais aussi lesquels, parmi les infracteurs, deviendront les plus persistants. 


\subsection{L'ÉCHEC SCOLAIRE}

Nous avons déjà souligné la conclusion de Loeber et Dishion (1983) à l'effet qu'une piètre performance scolaire (y compris le manque d'intelligence et l'école buissonnière) était un important signal précoce de la délinquance. Les études longitudinales ont toujours démontré la relation entre les deux phénomènes mais n'ont pas prouvé l'antériorité de l'échec scolaire par rapport à la délinquance. Par exemple, dans leur recherche longitudinale dans l'Oregon, Polk et al. (1981) ont trouvé que l'échec scolaire (mesuré par la moyenne des notes) était étroitement relié à la délinquance juvénile aussi bien qu'adulte, ce que Kelly (1980) considérait comme le résultat majeur de cette étude.

Dans une autre étude du genre, portant sur deux cents garçons noirs, Robins et Hill (1966) ont rapporté que l'absentéisme non motivé et le retard scolaire étaient reliés à la délinquance officielle précoce (quinze ans). Ils ont montré comment la probabilité de récidive augmentait avec l'école buissonnière, le retard scolaire et la faiblesse du statut socio-économique (mesuré par le prestige du métier des parents ou gardiens). En l'absence de ces facteurs, seulement $3 \%$ sont devenus délinquants, tandis qu'en présence des trois combinés, le taux grimpa à $36 \%$. Dans sa relance de jeunes sélectionnés lors de leur première comparution en cour juvénile, Meade (1973) a aussi mis en lumière l'échec scolaire comme un des meilleurs prédicteurs indépendants de la récidive.

Dans l'étude de cohorte de Philadelphie, Wolfgang et al. (1972) ont constaté que les mesures d'intelligence et de réussite de la première à la sixième année (six à douze ans) étaient en relation très significative avec la délinquance juvénile officielle. Ils ont conclu que ces résultats reflétaient les différences raciales quant à la délinquance puisque les gens de couleur avaient tendance à être peu intelligents, à connaître peu de succès et à se faire arrêter. Toutefois, en réanalysant les mêmes données, Jensen (1976) a montré que l'absence de réussite était plus fortement associée à la délinquance que ne l'était la race. Wolfgang et al. ont fait la découverte, non dénuée d'intérêt, que leurs délinquants chroniques avaient un niveau d'intelligence et de succès bien plus bas que les non-chroniques.

West et Farrington (1973), dans la recherche de Londres, ont trouvé que la faiblesse de l'intelligence (fortement reliée à 
d'autres mesures d'échec scolaire en bas âge) était parmi les meilleurs prédicteurs indépendants de la déclaration de culpabilité en cour juvénile. Qui plus est, la lenteur intellectuelle était tout particulièrement le fait des récidivistes et des enfants les plus précocement trouvés coupables de délit (dix à treize ans). Le manque d'intelligence et de succès permettaient de prédire la délinquance juvénile révélée autant qu'officielle (Farrington, 1979a). De plus, l'école buissonnière était un des principaux prédicteurs indépendants de la commission de délits à l'âge adulte (Farrington, 1985b), et le manque de succès entre huit et dix ans s'avérait des plus discriminant, non seulement entre les personnes trouvées coupables ou non mais aussi entre les formes chroniques ou non de l'engagement délinquant (Blumstein et al., 1985).

\subsection{LE GROUPE DE PAIRS}

Dans leur recherche longitudinale nationale américaine, Elliott et al. (1985) ont conclu que le fait de fréquenter des pairs délinquants était la principale cause immédiate de délinquance. Cette conclusion était essentiellement tirée d'analyses qui démontraient une étroite corrélation indépendante entre le fait d'avoir des amis délinquants et la délinquance révélée. Le principal problème d'interprétation est ici que, la plupart des actes délinquants étant commis en groupe, il est à peu près inévitable que leurs auteurs aient des amis délinquants. Autrement dit, le fait d'avouer des actes délinquants et la fréquentation de délinquants fourniraient deux mesures d'un même construit théorique qui est le comportement délinquant. Il n'a jamais été démontré dans la littérature que le fait de s'associer avec des délinquants précède ou facilite la commission de délits, bien que ce soit fort possible.

Dans l'étude longitudinale de Londres, on n'a pas mesuré la fréquentation d'amis délinquants avant quatorze ans, de sorte qu'elle n'a pas été explorée en tant que signal de délinquance en général, ni de délinquance chronique. Toutefois, ce facteur s'est avéré un bon prédicteur indépendant de la condamnation comme jeune adulte (Farrington, 1985b). Aussi, les récidivistes de dix-neuf ans qui avaient abandonné la délinquance se distinguaient de ceux qui poursuivaient dans cette voie par une plus grande tendance à délaisser leur groupe d'amis mâles. Qui plus est, dans les propos spontanés des jeunes, il apparaissait que, 
dans leurs propres perceptions, le retrait d'un groupe de pairs délinquants était un important facteur de l'abandon de la délinquance (West et Farrington, 1977). Donc, la persistance à fréquenter les amis délinquants peut être un élément capital du maintien de l'agir délinquant chez les jeunes adultes.

Nombre d'études ont montré que l'impopularité dans le jeune âge est un élément précurseur de l'agir délinquant. Ce fut démontré par Conger et Miller (1966) dans leur étude longitudinale rétrospective, ainsi que dans les vastes études prospectives de Roff et al. (1972) et de Roff et Wirt (1984). Dans l'étude longitudinale londonienne, l'impopularité entre huit et dix ans était un élément prédicteur de la délinquance adulte, mais non juvénile (Farrington, 1971a). L'impopularité des délinquants potentiels restreint probablement leur habileté à influencer la majorité des jeunes.

\section{LES INFLUENCES PROTECTRICES}

Dans la section précédente, on a analysé les principaux signaux précoces de l'agir délinquant. Surgit alors la question suivante : pourquoi certains enfants élevés dans des conditions typiquement criminogènes demeurent-ils à l'écart de la délinquance? Werner et Smith (1982) ont essayé de répondre à cette question dans leur étude longitudinale de sept cents enfants d'Hawaï, dont $15 \%$ environ se sont adonnés à une délinquance (officielle) grave et/ou persistante. Dans cette recherche, il est ressorti comme principaux antécédents de l'agir délinquant : le stress périnatal, les malformations congénitales, un bas niveau de vie (souvent la pauvreté chronique), la piètre éducation de la mère, le manque de stabilité de la famille, une carence intellectuelle et le fait d'avoir été mis en classe de rattrapage, le tout vers dix ans.

Werner et Smith ont trouvé que le meilleur point de démarcation entre les sujets à haut risque et les autres étaient la présence de quatre des facteurs énumérés ou plus. Ensuite, ils ont comparé soixante-douze "résistants», soit des enfants qui, tout en présentant le risque, n'avaient eu aucun problème sérieux d'apprentissage ou de comportement dans l'enfance ni de délinquance officielle à l'adolescence, avec les enfants à risque, du même âge et du même sexe, qui avaient un dossier de délinquance lourde et/ou persistante à l'adolescence. Werner 
(1983) a trouvé que les enfants résistants avaient tendance : à être premiers-nés, actifs et affectueux dans leur tendre enfance; à appartenir à des familles sans discorde ou presque, où étaient présents les deux parents et où la mère ne travaillait pas; à avoir été des bébés choyés et intelligents; à bien s'exprimer verbalement; et à avoir une grande estime de soi à l'adolescence. Ces résultats nous suggèrent que, tout comme la séparation d'avec les parents, les conflits entre les parents et le manque d'intelligence peuvent être des éléments précurseurs de l'agir délinquant, leurs contraires peuvent avoir un rôle préventif. Des chercheurs ont aussi porté attention à la petite dimension de la faille (conséquemment, au degré élevé d'attention donné à l'enfant) comme facteur préventif. Une famille nombreuse a souvent été identifiée comme facteur relié à la délinquance. West et Farrington (1973) ont trouvé que les garçons qui avaient à dix ans au moins quatre frères et sœurs avaient plus tendance à se rendre coupables de délits juvéniles; et Farrington (1979a) a montré que la grande dimension de la famille aidait à prédire la délinquance, révélée comme officielle.

Dans l'étude longitudinale de Londres, 63 garçons ont été identifiés comme vulnérables à la délinquance en cela qu'ils présentaient au moins trois des cinq principaux facteurs criminogènes (parents déclarés coupables, famille à faible revenu, famille nombreuse, méthodes éducatives déficientes et faible intelligence : voir West et Farrington, 1973). Sur les 63, 31 ont été trouvés coupables comme mineurs et huit comme jeunes adultes. Toutefois, huit autres n'avaient pas de délinquance officielle et une légère délinquance révélée. Ces derniers, souvent des garçons sages issus de mauvais milieux, présentaient tous des problèmes autres que la délinquance. On les décrivait comme anxieux, nerveux, toujours tendus, obsessifs, timides, silencieux, suspicieux, inhibés ou apathiques. En d'autres termes, les garçons issus de milieux criminogènes qui ne devenaient pas délinquants avaient tendance à devenir nerveux et renfermés et il est envisageable que la délinquance et le retrait nerveux soient les deux réactions possibles à un milieu d'origine stressant.

Une étude ultérieure des garçons sages issus de mauvais milieux, décrite par West (1982), confirme en gros ces conclusions déprimantes. De 397 hommes toujours vivants et résidant au Royaume-Uni à vingt-deux ans, 54 furent identifiés comme 
ayant un passé sérieusement carencé, c'est-à-dire qu'ils avaient grandi dans une famille criminelle et avaient expérimenté, dans leur jeune âge, au moins deux des situations suivantes : illégitimité, famille prestataire de l'aide sociale, sous les soins des autorités locales, un foyer brisé par une autre cause que le décès, résidant dans un bas quartier, d'une nombreuse famille. Parmi ces hommes, seulement dix-huit n'avaient pas été condamnés et on a tenté de les interviewer autour de vingt-quatre ans. En comparaison avec ceux qui n'avaient pas non plus été condamnés mais qui provenaient de milieux non carencés, ces sujets se caractérisaient par des échecs sociaux comme le chômage chronique, le découragement, la saleté ou un logement surpeuplé, et par l'isolement social. Il y avait très peu de réussites.

Malgré tout, il existe des événements dans la vie qui semblent favoriser une décroissance de l'agir délinquant, dont deux ont été décrits par West (1982). Les hommes qui épousent des femmes sans dossier criminel semblent devenir moins criminels par la suite, si on les compare à ceux qui ne se marient pas et à ceux qui épousent des femmes criminelles (ces derniers semblent accentuer leur criminalité). De plus, déménager hors de Londres amène une diminution de la délinquance officielle et révélée, peut-être en favorisant une rupture bénéfique avec les amis criminels. Un autre exemple, Elliott (1966) et Elliott et Voss (1974) ont montré que la délinquance officielle et révélée diminuaient suite à l'abandon scolaire. Toutefois, aucun de ces résultats ne permet d'identifier les facteurs protecteurs qui neutralisent les éléments favorables à l'agir délinquant.

En somme, on connaît peu de choses sur les influences protectrices, mis à part les contraires des signaux de la délinquance.

\section{CONCLUSION}

Alors qu'on connait passablement bien les signes avantcoureurs précoces de la délinquance, on connaît assez peu de la délinquance à haute fréquence. Les recherches longitudinales actuelles et futures devraient plus explicitement tenter de prédire qui seront les délinquants prolifiques (parmi l'ensemble des auteurs de délits et parmi la population en général) et les auteurs de délits en général. Ces études devraient également chercher à identifier les facteurs susceptibles de modifier le parcours des 
jeunes à risques élevés de délinquance chronique. Pour le moment, à peu près la seule étude pertinente sur les signaux précoces de la délinquance chronique est celle de Blumstein et al. (1985). Ils ont trouvé que les troubles précoces de comportement, la condamnation des parents et de la fratrie, le manque d'intelligence et l'insuccès aidaient à prédire la délinquance en général et plus spécifiquement la délinquance chronique. Un bas revenu familial s'avérait aussi un facteur de prédiction mais pas indépendamment des autres facteurs; et de mauvais comportements éducatifs des parents, de même que leur séparation par suite de mésentente, étaient prédicteurs de la délinquance, mais pas de sa chronicité.

Dans l'étude londonienne, il fut étonnant de constater à quel point l'agir délinquant chronique était prévisible. On a construit une échelle de prédiction de l'agir délinquant en sept points à partir du comportement-problème (trois mesures), de la carence sociale, la condamnation des parents, la pauvreté de l'intelligence et le mauvais comportement éducatif, tous entre huit et dix ans. L'échelle fut construite à partir de la moitié d'un échantillon tiré au hasard et validé avec l'autre moitié (pour les détails, se référer à Farrington, 1985a). Les 55 jeunes ayant obtenu un score d'au moins quatre sur sept comprenaient la majorité (15 sur 23 ) des délinquants chroniques, c'est-à-dire ayant commis six délits ou plus, 22 des 109 délinquants non chroniques et 18 des 265 jeunes n'ayant jamais été reconnus coupables.

Au-delà de notre attente, on a pu identifier les délinquants chroniques au moment où ils furent trouvés coupables pour la première fois, grâce à l'information disponible lorsqu'ils avaient dix ans. Toutefois, il y a peu d'intérêt à prédire qui seront les délinquants chroniques si on ne sait pas comment les traiter. Et pour trouver le meilleur traitement, il faut connaitre les causes de la délinquance à haute fréquence et on n'y est pas encore. À l'avenir, les recherches longitudinales sur les signaux précoces devraient comporter de fréquentes entrevues avec les sujets, de sorte qu'on puisse identifier les événements critiques qui engendrent un accroissement de l'agir délinquant.

En plus des recherches longitudinales, on a besoin d'études expérimentales pour explorer systématiquement l'efficacité des mesures visant à neutraliser les signaux de la délinquance chro- 
nique. Par exemple, il serait possible de donner un supplément de revenu aux familles les plus pauvres ou de leur donner de meilleures conditions de vie; on pourrait faire bénéficier les élèves aux prises avec des échecs scolaires d'un tutorat spécial ou de programmes initialement enrichis de manière à améliorer leurs performances; et il y aurait lieu de fournir aux parents et de tester un apprentissage de techniques d'éducation ou de résolution de conflits. Le succès ou l'insuccès de ces expériences permettrait aussi de mettre en lumière jusqu'où ces éléments précurseurs étaient des causes plutôt que de simples facteurs associés à l'agir délinquant. On a aussi besoin de recherches expérimentales pour connaître l'efficacité des mesures judiciaires appliquées aux auteurs de délits virtuellement chroniques, lors de leur premier contact à la Cour.

Vu que les années 70 ont mené à la découverte majeure de l'agir délinquant chronique et que les années 80 sont celles de l'accumulation d'un savoir sur ses signaux précoces, on doit planifier les recherches des années 90 pour qu'elles mènent à la découverte de méthodes efficaces pour réduire la délinquance chronique. Tout comme les délinquants chroniques contribuent d'une manière disproportionnée au problème du crime, ces recherches pourraient le diminuer d'autant.

\section{RÉFÉRENCES}

BLUMSTEIN, Alfred, David P. FARRINGTON et Soumyo MOITRA (1985), «Delinquency Careers : Innocents, Desisters, and Persisters", dans Michael Tonry and Norval Morris (édit.), Crime and Justice, vol. 6, Chicago University of Chicago Press.

BLUMSTEIN, Alfred et Soumyo MOITRA (1980), "The Identification of Career Criminals from Chronic Offenders in a Cohort", Law and Policy Quarterly $2: 321-334$.

CHAIKEN, Jan M. et Marcia R. CHAIKEN (1982), Varieties of Criminal Behavior, Santa Monica, CA, Rand Corporation.

CONGER, James J. et Walter C. MILLER (1966), Personality, Social Class, and Delinquency, New York, Wiley.

CRAIG, Maude M. et Selma J. GLICK (1968), "School Behavior Related to Later Delinquency and Non-Delinquency», Criminologica 5 : 17-27.

DOUGLAS, James W.B., J.M. ROSS, Walter A. HAMMOND et D. Glenn MULLIGAN (1966), «Delinquency and social Class», British Journal of Criminology $6: 294-302$. 
DUNFORD, Franklin et Delbert S. ELLIOTT (1984), «Identifying Career Offenders using Self-Reported Data», Journal of Research in Crime and Delinquency $21: 1$ 57-86.

ELLIOTT, Delbert S. (1966), "Delinquency, School Attendance, and Dropout», Social Problems 13 : 307-314.

ELLIOTT, Delbert S., David HUIZINGA et Suzanne S. AGETON (1985), Explaining Delinquency and Drug Use, Beverly Hills, CA, Sage.

ELLIOTT, Delbert S. et Harwin L. VOSS (1974), Delinquency and Dropout, Lexington, MA, Heath.

EMSMINGER, Margaret E., Sheppard G. KELLAM et B.R. RUBIN (1983), "School and Family Origins of Delinquency», dans Katherine T. Van Dusen and Sarnoff A. Mednick (édit.) Prospective Studies of Crime and Delinquency, Boston, Kluwer-Nijhoff.

FARRINGTON, David P. (1978), "The Family Backgrounds of Agressive Youths", dans Lionel Hersov, Michael Berger and David Shaffer (édit.), Aggression and Antisocial Behavior in Childhood and Adolescence. Oxford, Pergamon.

FARRINGTON, David P. (1979a), «Environmental Stress, Delinquent Behavior, and Convictions», dans Irwin G. Sarason and Charles D. Spielberger (édit.), Stress and Anxiety, vol. 6, Washington, DC, Hemisphere.

FARRINGTON, David P. (1979b), «Longitudinal Research on Crime and Delinquency», dans Norval Morris and Michael Tonry (édit.), Crime and Justice, vol. 1, Chicago, University of Chicago Press.

FARRINGTON, David P. (1983), «Offending from 10 to 25 Years of Age», dans Katherine T. Van Dusen and Sarnoff A. Mednick (édit.), Prospective Studies of Crime and Delinquency, Boston, Kluwer-Nijhoff.

FARRINGTON, David P. (1985a), «Predicting Self-Reported and Official Delinquency», dans David P. Farrington and Roger Tarling (édit.), Prediction in Criminology, Albany, NY, State University of New York Press.

FARRINGTON, David P. (1985b), «Stepping Stones ot Adult Criminal Careers», dans Dan Olweus, Jack Block and Marian R. Yarrow (édit.), Development of Antisocial and Prosocial Behavior, New York, Academic Press.

GREENWOOD, Peter W. et Allan ABRAHAMSE (1982), Selective Incapacitation, Santa Monica, CA, Rand Corporation.

HOME OFFICE (1985), Criminal Careers of Those Born in 1953, 1958, and 1963, London, Home Office Statistical Bulletin $7 / 85$.

JENSEN, Gary F. (1976), «Race, Achievement, and Delinquency : A Further Look at Delinquency in a Birth Cohort», American Journal of Sociology $82: 379-387$.

KELLY, Delos H. (1980), «The Educational Experience and Evolving Delinquent Careers», dans David Shichor and Delos H. Kelly (édit.), Critical Issues in Juvenile Delinquency, Lexington, MA, Heath.

LOEBER, Rolf et Thomas DISHION (1983), "Early Predictors of Male Delinquency : A Review», Psychological Bulletin 94 : 68-99. 
McCORD, Joan (1977), «A Comparative Study of Two Generations of Native Americans", dans Robert F. Meier (édit.), Theory in Criminology, Beverly Hills, CA, Sage.

McCORD, Joan (1979), «Some Child-Rearing Antecedents of Criminal Behavior in Adult Men", Journal of Personality and Social Psychology 37: 1477-1486.

McCORD, Joan (1982), "A Longitudinal View of the Relationship Between Paternal Absence and Crime», dans John Gunn and David P. Farrington (édit.), Abnormal Offenders, Delinquency, and the Criminal Justice System, Chicester, Wiley.

MAGNUSSON, David, A. DUNER et G. ZETTERBLOM (1975), Adjustment : A Longitudinal Study, Stockholm, Almqvist and Wiksell.

MEADE, Anthony (1973) «Seriousness of Delinquency, the Adjudicative Decision, and Recidivism - A Longitudinal Configuration Analysis», Journal of Criminal Law and Criminology $64: 478-485$.

MULLIGAN, Glenn, James W.B. DOUGLAS, Walter A. HAMMOND et Jack TIZARD (1963), "Delinquency and Symptoms of Maladjustment», Proceedings of the Royal Society of Medicine 56 : 1083-1086.

PETERSON, Mark A., Harriet B. BRAIKER et Suzanne M. POLICH (1981), Who Commits Crimes? Cambridge, MA, Oelgeschlager, Gunn and Hain.

POLK, Kenneth, C. ALDER, G. BAZEMORE, G. BLAKE, S. CORDRAY, G. COVENTRY, J. GALVIN et M. TEMPLE (1981), Becoming Adult, Final Report to the National Institute of Mental Health, Washington, DC.

POWER, Michael J., P.M. ASH, E. SHOENBERG et E.C. SIREY (1974), «Delinquency and the Family», British Journal of Social Work $4: 13-38$.

ROBINS, Lee N. (1966), Deviant Children Grown Up, Baltimore, Williams and Wilkins.

ROBINS, Lee N. (1979), «Study Childhood Predictors of Adult Outcomes : Replications from Longitudinal Studies", dans James E. Barrett, Robert M. Rose, and Gerald L. Klerman (édit.), Stress and Mental Disorder, New York, Raven Press.

ROBINS, Lee N. (1983), "Continuities and Discontinuities in the Psychiatric Disorders of Children", dans David E. Mechanic (édit.), Handbook of Health, Health Care and the Health Professions, New York, Free Press.

ROBINS, Lee N. et Shirley Y. HILL (1966), "Assessing the Contributions of Family Structure, Class, and Peer Groups to Juvenile Delinquency», Journal of Criminal Law, Criminology, and Police Science 57 : 325-334.

ROBINS, Lee N, et Kathryn S. RATCLIFF (1980), "Childhood Conduct Disorders and Later Arrest», dans Paula J. Clayton and John K. Wing (édit.), The Social Consequences of Psychiatric Illness, New York, Brunner/Mazel.

ROBINS, Lee N., Patricia A. WEST et Barbara L. HERJANIC (1975), «Arrests and Delinquency in Two Generations : A Study of Black Urban Families and their Children», Journal of Child Psychology and Psychiatry $16: 125-140$. 
ROFF, James D. et Robert D. WIRT (1984), «Childhood Aggression and Social Adjustment as Antecedents of Delinquency», Journal of Abnormal Child Psychology 12 : 111-126.

ROFF, Merrill, S.B. SELLS et M.M. GOLDFN (1972), Social Adjustment and Personality Development in Children, Minneapolis, University of Minnesota Press.

SHANNON, Lyle W. (1978), "A Longitudinal Study of Delinquency and Crime», dans Charles Wellford (édit.), Quantitative Studies in Criminology, Beverly Hills, CA, Sage.

THORNBERRY, Terence P. et Margaret FARNWORTH (1982), "Social correlates of Criminal Involvement : Further Evidence on the Relationship Between Social Status and Criminal Behavior», American Sociological Review 47 : 505-518.

VAN DUSEN, Katherine T., Sarnoff A. MEDNICK, William F. GABRIELLI et Barry HUTCHINGS (1983), "Social Class and Crime in an Adoption Cohort", Journal of Criminal Law and Criminology $74: 249-269$.

WADSWORTH, Michael (1979), Roots of Delinquency, London, Martin Robertson.

WERNER, Emmy E. (1983), «Vulnerability and Resiliency among Children at Risk for Delinquency : A Longitudinal Study", Paper presented at the Annual Meeting of the American Society of Criminology, Denver, CO.

WERNER, Emmy E. et Ruth S. SMITH (1982), Vulnerable but Invincible, New York, McGraw-Hill.

WEST, Donald J. (1982), Delinquency : Its Roots, Careers, and Prospects, London, Heinemann.

WEST, Donald J. et David P. FARRINGTON (1973), Who Becomes Delinquent? London, Heinemann.

WEST, Donald J. et David P. FARRINGTON (1977), The Delinquent Way of Life, London, Heinemann.

WOLFGANG, Marvin E., Robert M. FIGLIO et Thorsten SELLIN (1972), Delinquency in a Birth Cohort, Chicago, University of Chicago Press.

WOLFGANG, Marvin E. et Paul E. TRACY (1982), "The 1945 and 1958 Birth Cohorts : A Comparison of the Prevalence, Incidence, and Severity of Delinquent Behavior», Paper Presented at Conference on «Public Danger, Dangerous Offenders, and the Criminal Justice System». 